\title{
OBSERVATIONS \\ SUR LE DÉVELOPPEMENT EMBRYONNAIRE \\ ET LARVAIRE DE RANA TEMPORARIA L. \\ [Batracien, Anoure]
}

par E. et M.-L. ANGelier.

Le développement embryonnaire et larvaire de Rana temporaria L. a fait l'objet de travaux nombreux; peu cependant abordent le problème sous l'aspect écologique.

Le rôle des sels dissous sur la croissance et la métamorphose des têtards a été envisagé par certains auteurs.

Après quelques travaux anciens, A. I. IR Iкнмоvітсн [1947], J. P. VAN der Eeckhoudt [1949], H. Kauri [1955], A. Beer [1956], H. Doetsch [1958], E. Rühmekorf [1958], R. Guyétant [1964, 1966] ont montré récemment l'effet variable de la lumière sur le développement de diverses espèceś d'Anoures.

J. S. Mikulski [1938], J. A. Moore [1939, 1951], R. Doyglas [1948], F. Kopsch [1952], P. Roth [1955], E. Balcells [1956], donnent des indications sur le développement, - surtout embryonnaire, - de Rana temporaria en fonction de la température.

Des recherches sur les lacs du Massif du Néouvielle (HautesPyrénées) nous ont permis d'observer le développement larvaire de quelques espèces de Batraciens en fonction de l'altitude, - en fait, de la température.

La durée de la vie larvaire s'accroît considérablement en haute montagne pour les espèces vivant habituellement à basse altitude. A $2100 \mathrm{~m}$, la reproduction de Triturus helveticus se déroule en août, et la vie larvaire dure plus d'un an [E. AngeliER, 1961]. La vie larvaire peut durer une vingtaine d'années, à $2400 \mathrm{~m}$, chez Alytes obstetricans [E. et M. $\lrcorner$ L. ANGELIER, 1964] ; d'après E. RüHMEKORF [1958], la température préférée de la larve se situerait entre 21,9 et $25,3^{\circ} \mathrm{C}$. 


\section{1. - REPRODUCTION ET DEVELOPPEMENT DE RANA TEMPORARIA DANS LE MASSIF DU NEOUVIELLE (HAUTES-PYRENEES).}

Le problème du développement larvaire de Rana temporaria dans les lacs de haute altitude apparaît plus complexe que celui de Triturus helveticus ou Alytes obstetricans. Il s'agit d'une espèce sténotherme; selon E. BALcells [1956], sa limite sud de répartition ne dépasse pas l'isotherme de $21^{\circ} \mathrm{C}$ en juillet. Dans le Sud-Ouest de la France, elle ne vit généralement qu'au-dessus de $1000 \mathrm{~m}$, — et on la rencontre jusqu'à $2900 \mathrm{~m}$ d'altitude.

\section{1. - Reproduction.}

C. Combes [1967] a publié une série d'observations sur la biologie de Rana temporaria dans la partie orientale des Pyrénées. La ponte est d'autant plus tardive que l'altitude des biotopes est plus élevée. Le décalage peut atteindre 3 mois entre les points les plus bas étudiés par cet auteur $(1100 \mathrm{~m})$ et les points les plus élevés $(2300 \mathrm{~m})$. Il existe également des variations, d'une année à l'autre, en fonction des conditions météorologiques, - et notamment de l'enneigement.

Dans les Pyrénées centrales, la ponte se déroule depuis le début avril (lac d'Oô, Haute-Garonne, à $1500 \mathrm{~m}$ ) jusqu'en juin (lac Supérieur d'Estihère, Hautes-Pyrénées, à $2330 \mathrm{~m}$ ). Elle paraît suivre le dégel des mares et des lacs.

La durée de la période de reproduction, dans chaque station, est très courte (quelques jours). La ponte a lieu dans le $1^{\text {er }}$ point d'eau libéré de la glace. Ainsi, près de la Station Biologique du Lac d'Orédon, une petite mare de quelques $\mathrm{m}^{2}$ se forme précocement chaque année, lors de la fonte des neiges. Rana temporaria pond dès que la mare apparaît. Or, les tétards meurent toujours avant la métamorphose, car la mare se dessèche très rapidement.

Lorsque l'enneigement est faible, et que les mares de fonte des neiges n'ont qu'une durée éphémère, le nombre de populations de têtards mourant ainsi avant de se métamorphoser est considérable.

Le lieu d'hibernation et le lieu de ponte sont évidemment distincts lorsque ce dernier est à sec en automne, et que la grenouille ne peut s'enfouir dans la vase. Ils peuvent être également distincts dans les eaux permanentes. Au lac Inférieur d'Estibère $(2120 \mathrm{~m})$, nous avons vu les couples de Rana temporaria traverser toute la surface encore gelée du lac pour venir pondre dans les quelques $\mathbf{m}^{2}$ d'eau libre, sur la rive Sud. Ils n'avaient pas hiberné dans le lac. L'absence de points d'eau sur la rive Nord du lac suggère la possibilité d'une hibernation à terre. 


\section{2. - Durée de la vie larvaire en fonction de l'altitude.}

En 1957 [E. ANGelier, 1961], les oufs de Rana temporaria ont éclos le $1^{\text {er }}$ juillet dans le lac Supérieur d'Estibère $(2330 \mathrm{~m})$. La métamorphose commença dès le 25 août, et la population entière devint adulte en une semaine environ. Le développement larvaire a duré moins de 2 mois. La température du lac ne dépasse par $16-18^{\circ} \mathrm{C}$ en juillet-août.

Dans le lac Inférieur d'Estibère $(2120 \mathrm{~m})$, l'éclosion des œufs a été beaucoup plus précoce, - aux environs du 20 mai. Dès le 10 juillet, les têtards avaient les pattes postérieures bien développées, en flexion comme chez l'adulte (stade $\mathbf{K}$ de l'échelle de Kollmann). A cette époque déjà, et durant tout le mois d'août, la température du lac atteignait 21 à $23^{\circ} \mathrm{C}$. On observe des têtards non métamorphosés jusqu'au début septembre. La vie larvaire a duré 3 mois $\frac{1}{2}$.

Dans le lac d'Anglade, un peu au-dessus du précédent (2 $170 \mathrm{~m})$, les œufs ont éclos le $1^{\text {er }}$ juin, et les têtards les plus tardifs se sont métamorphosés vers le 25 août. La température du lac n'a pratiquement pas dépassé $20-21^{\circ} \mathrm{C}$ en juillet-août.

Dans les mares temporaires de très faible profondeur, l'amplitude quotidienne de la température est considérable. Nous avons noté des températures minimales de 8 à $10^{\circ} \mathrm{C}$, et des températures maximales de 30 à $32^{\circ} \mathrm{C}$, en juin, dans une mare à $1900 \mathrm{~m}$ d'altitude, près de la Station Biologique du Lac d'Orédon. Le développement larvaire semble accéléré, puisque les têtards éclos le $\mathbf{1}^{\mathrm{er}}$ juin se métamorphosaient déjà le 25 juin.

La longueur des jeunes grenouilles, de la pointe du museau à l'extrémité du tronc, varie considérablement: de $11 \mathrm{~mm}$ (lorsque la vie larvaire dure 25 jours) à $17 \mathrm{~mm}$ (lac Supérieur d'Estibère...).

Nous avions donné [F. ANGelier, 1961] une interprétation de ces observations. Contrairement à Triturus helveticus et Alytes obstetricans, Rana temporaria est une espèce sténotherme. Les températures constamment élevées détermineraient un allongement de la vie larvaire, par inhibition de la métamorphose (lac Inférieur). Les températures alternées (é!evées le jour, basses la nuit) détermineraient par contre une accélération du développement (mares de faibles profondeur).

Des observations ultérieures, plus minutieuses, ne confirmèrent pas cette interprétation.

Dans le lac Inférieur d'Estibère, les premières métamorphoses se déroulent dès la fin du mois de juillet, et les jeunes grenouilles sont de petite taille. Durant tout le mois d'août, des têtards vont se métamorphoser, et la population toute entière sera devenue adulte au début septembre. La longueur des grenouilles les plus tardives atteint $17 \mathrm{~mm}$ en moyenne. 
Dans les eaux à température élevée, ce n'est donc pas véritablement une inhibition de la métamorphose qu'on observe, mais plutôt un étalement de celle-ci sur une très longue période (plus d'un mois). Durant les journées ensoleillées, les têtards viennent se concentrer sur les fonds de quelques centimètres, le long de la rive, de sorte qu'ils sont soumis à des températures encore plus élevées que celle du lac lui-même $\left(25\right.$ à $30^{\circ} \mathrm{C}$, Iorsque le lac atteint $22-23^{\circ} \mathrm{C}$ ).

Dans les mares temporaires de faible profondeur, le phénomène d'étalement de la métamorphose est identique. Mais, par suite de - l'assèchement rapide, les têtards les plus précoces ont seuls le temps de devenir adultes. Les autres meurent, rassemblés en quelques points où l'eau a persisté un peu plus longtemps.

Le fait important, c'est l'homogénéité du développement larvaire dans les lacs élevés du Massif du Néouvielle, et au contraire l'étalement dans le temps de la métamorphose dans les lacs les plus bas.

Afin de comprendre le rôle exact de la température sur le développement de Rana temporaria, nous avons réalisé, de 1964 à 1967, des élevages au laboratoire.

Nous avons utilisé, pour ces expériences, des pontes provenant du lac d'Oô (Haute-Garonne; altitude : $1500 \mathrm{~m}$ ), de l'étang des Nymphes ou d'une mare proche, près de la Station Biologique du Lac d'Orédon (Hautes-Pyrénées; altitude : $1900 \mathrm{~m}$ ), ou bien de mares temporaires situées près du lac d'Aumar (Hautes-Pyrénées; altitude : $2200 \mathrm{~m}$ ).

Il s'agit, dans toutes les stations, de la race différenciée de Rana temporaria, déjà signalée dans les Pyrénées par L. GaLLien [1941]. Les coupes effectuées dans les gonades des 2 sexes, au moment de la métamorphose, montrent :

- chez les , la structure ovarienne très nette, avec les poches ovariennes correspondant aux cordons médullaires qui se sont creusés pour former le sac ovarien;

- chez les ô, les testicules, dont les cordons médullaires, massifs, enrobent l'ensemble des spermatogonies.

2. - INFLUENCE DE LA TEMPERATURE SUR LE DEVELOPPEMENT EMBRYONNAIRE DE RANA TEMPORARIA.

Les expériences ont été effectuées en 1967, à partir d'une seule ponte recueillie dans une mare proche de l'étang des Nymphes, le 21 mai 1967. La mare n'était dégelée que sur une surface de $1 \mathrm{~m}^{2}$ environ, près d'un rocher, et la température de l'eau était 
de $0,5^{\circ} \mathrm{C}$ à $11 \mathrm{~h}$. Les pontes, au nombres d'une dizaine, dataient de la nuit précédente.

Le 22 mai, les œufs sont répartis dans 8 aquariums, aux températures respectives de $7,10,13,16,19,22,25$ et $28^{\circ} \mathrm{C}\left( \pm 0,5^{\circ} \mathrm{C}\right)$.

Les résultats n'ont évidemment qu'une valeur relative. Les pontes ont été transportées dans des bouteilles isolantes, contenant un mélange d'eau et de neige; il s'est néanmoins écoulé $36 \mathrm{~h}$ entre la ponte et la mise en élevage.

2.1. - Durée du développement embryonnaire en fonction de la température.

La durée du développement embryonnaire varie entre 3 jours, - à $25^{\circ} \mathrm{C}$, - et 20 jours, - à $7^{\circ} \mathrm{C}$. La courbe représentant cette durée en fonction de la température est une hyperbole (fig. 1).

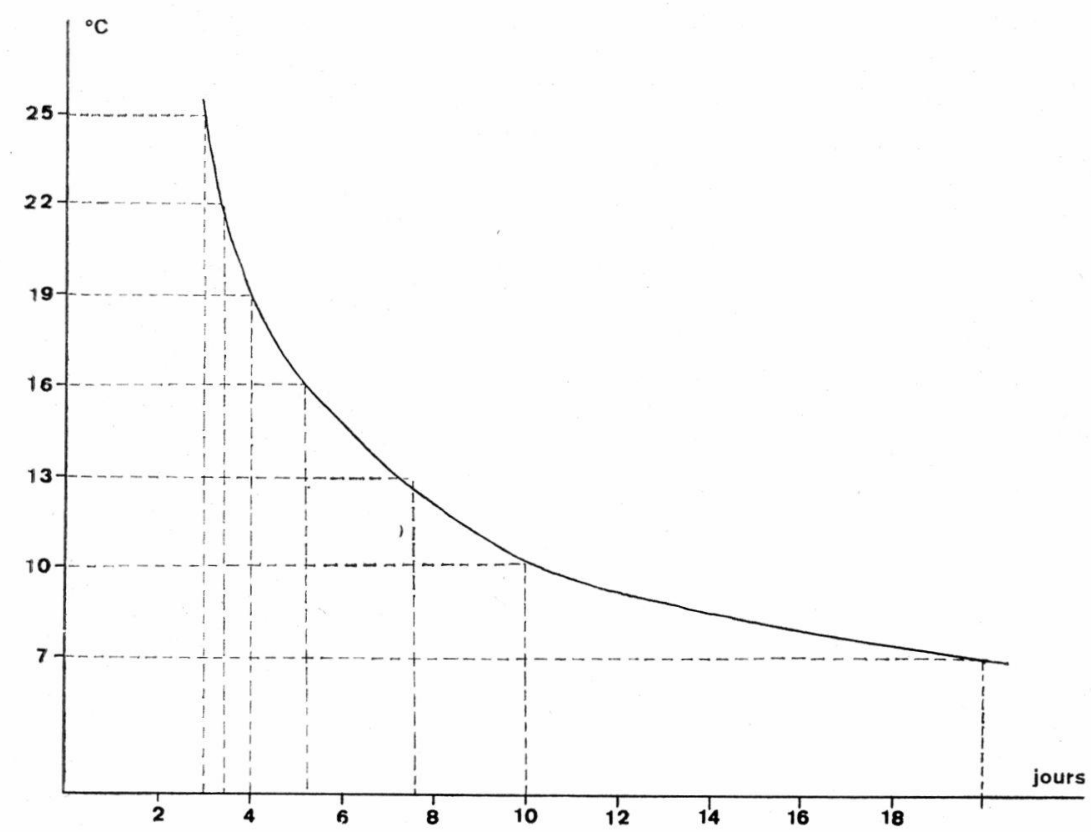

Fig. 1. - Durée du développement embryonnaire de Rana temporaria en fonction de la température.

\section{2. - Température létale.}

A $28^{\circ} \mathrm{C}$, quelques larves ont éclos au bout de $48 \mathrm{~h}$, mais sont mortes très rapidement. La plupart des œufs ont commencé leur développement, mais n'ont pu atteindre l'éclosion. 
On peut considérer que $25-26^{\circ} \mathrm{C}$ constitue la température limite pour le développement embryonnaire de Rana temporaria.

\section{3. - Température seuil et Constante thermique.}

Nous n'avons pu expérimenter à des températures inférieures à $7^{\circ} \mathrm{C}$; la régulation précise des aquariums thermostatés devient impossible lorsque la température ambiante est élevée (fin du printemps et été).

On peut cependant calculer la température seuil, par l'intermédiaire de la Constante thermique. On sait que, pour une espèce donnée, le produit de la durée du développement, en jours $(\mathbf{N})$, par la température efficace ( $\mathrm{T}-\mathrm{T}$ seuil) est constant.

Nous pouvons tirer des données expérimentales :

$\mathrm{C}^{\mathrm{ce}}$ thermique $=\left(\mathrm{T}-\mathrm{T}_{\mathrm{s}}\right) \mathrm{N}$

$$
=\left(7-\mathrm{T}_{\mathrm{s}}\right) 20=\left(10-\mathrm{T}_{\mathrm{s}}\right) 10=\left(13-\mathrm{T}_{\mathrm{s}}\right) 7=\ldots
$$

A partir de ces égalités, nous pouvons connaître la température seuil, $-4^{\circ} \mathrm{C}$ - et la Constante thermique, -60 environ.

Le développement embryonnaire de Rana temporaria est donc possible, - d'après nos expériences, pour des températures comprises entre 4 et $25-26^{\circ} \mathrm{C}$, la Constante thermique étant de 60 .

Les températures limites correspondent à celles observées par J. S. Mikulski, 1938 (5 et $\left.26^{\circ} \mathrm{C}\right)$, R. Douglas, 1948 (3,3 et 24-25 ${ }^{\circ} \mathrm{C}$ ), J. A. Moore, $1951\left(5\right.$ et $25^{\circ} \mathrm{C}$ ) et E. BAlcells, $1956\left(4\right.$ et $24,6^{\circ} \mathrm{C}$ ).

Ces auteurs ne mentionnent pas la Constante thermique; mais les durées de développement aux diverses températures qu’ils indiquent permettent de la calculer.

D'après les observations de R. Douglas et E. Balcells, la Constante thermique est égale à 60 , ce qui confirme nos propres résultats.

Selon les données de J. A. Moore, clle serait de $\mathbf{5 0}$ seulement, ce qui correspond à un développement plus rapide.

Les expériences de J. S. Mikulski manquent probablement de précision; la Constante thermique varie en effet entre 44 et 63 , et n'a par conséquent aucune signification.

\section{3. - INFLUENCE DE LA TEMPERATURE SUR LE DEVE- LOPPEMENT LARVAIRE.}

\section{1. - Techniques d'élevage.}

Des élevages ont été réalisés chaque année, de 1964 à 1967 . Pour une série d'expérience données, nous avons utilisé systématiquement des œufs issus d'une même ponte. 
La température des aquariums était régulée à $\pm 0,5^{\circ} \mathrm{C}$. L'aération de l'eau était assurée par l'air comprimé; à partir de 1965, cette eau était constamment filtrée sur charbon.

L'eau était renouvelée tous les 3 jours, - les excréments et détritus aspirés chaque jour.

Nourriture. - Les têtards étaient nourris de salade cuite, toujours en excès; ils recevaient en outre, une fois par semaine, une poudre à base de farine de Poissons (Tetraphyll).

Nous n'avons pas cherché à obtenir des résultats rigoureux en valeur absolue, mais des résultats comparables, nous permettant de comprendre les phénomènes observés. C'est pourquoi les têtards issus d'une même ponte n'étaient mis en élevage qu'après l'operculation des branchies. Les pertes qui suivent l'éclosion des œufs ou celles qui peuvent résulter d'une manipulation trop précoce des têtards sont terminées. Nous avons pu ainsi conserver dans chaque aquarium un nombre d'individus à peu près constant du début à la fin des expériences : 40 têtards pour un aquarium contenant 17 litres d'eau.

\section{2. - Méthodes de mesure.}

Les têtards ont été mesurés régulièrement, à des intervalles variables suivant la vitesse de croissance (tous les 3 jours à température élevée; tous les 10 à 15 jours à $7^{\circ}$ et $10^{\circ} \mathrm{C}$ ).

Après anesthésie (au MS 222 Sandoz), ils étaient posés sur un papier millimétré paraffiné. On notait la longueur totale, la longueur du tronc (de la pointe du museau à l'anus), ainsi que le stade du développement.

La définition des différents stades correspond à celle de l'échelle de Kollmann, complétée par P. Roth [1955] :

Stade A : Aucune trace de pattes postérieures

Stade B : Présence de bourgeons blanchâtres, précédant l'apparition des pattes postérieures

Stade C : Pattes postérieures visibles, mais très petites

Stade D: Pattes postérieures plus grandes, mais presque droites et allongées sur la queue

Stade E : Pattes postérieures encore plus grandes, mais toujours allongées sur la queue

Stade F : Pattes postérieures en flexion, comme chez l'adulte

Stade G: Présence des bourgeons des pattes antérieures, nettement différenciés

Stade $\mathrm{H}$ : Présence des membres antérieurs

Stade I : Début de réduction de la queue

Stade $\mathbf{J}$ : Résorption complète de la queue. 


\section{3. - Durée de la vie larvaire en fonction de la température.}

Les expériences ont été effectuées en 1964 et 1965. En 1964, les têtards étaient issus d'une ponte unique, recueillie près du lac d'Oô (à $1500 \mathrm{~m}$ d'altitude), et mis en élevage le 24 avril, dès l'operculation des branchies.

6 aquariums étaient régulés respectivement à $7,10,13,16,19$ et $22^{\circ} \mathrm{C}$. L'éclairement était celui de la lumière du jour, les fenêtres de la salle d'élevage s'ouvrant au Sud-Est.

En 1965 , les aquariums étaient régulés à $20,22,24,26,28,30$, 31 et $32^{\circ} \mathrm{C}$. La ponte de Rana temporaria avait été récoltée dans une mare de fonte des neiges, à $2200 \mathrm{~m}$ d'altitude, près du lac d'Aumar. Les branchies des têtards étaient operculées le 5 juin, date du début des expériences.

Les conditions expérimentales n'étaient donc pas rigoureusement les mêmes en 1964 et 1965 . Les pontes venaient de 2 stations très différentes par l'altitude, et le développement des têtards s'est déroulé au printemps, - en 1964, - et à la fin du printemps-début de l'été en 1965.

La durée de la vie larvaire (à partir du stade $\mathbf{A}$, aux branchies operculées, jusqu'au stade $\mathbf{J}$ ) varie entre une vingtaine de jours $\left(\right.$ à $28^{\circ} \mathrm{C}$ ) et près d'un an $\frac{1}{2}$ (à $7^{\circ} \mathrm{C}$ ).

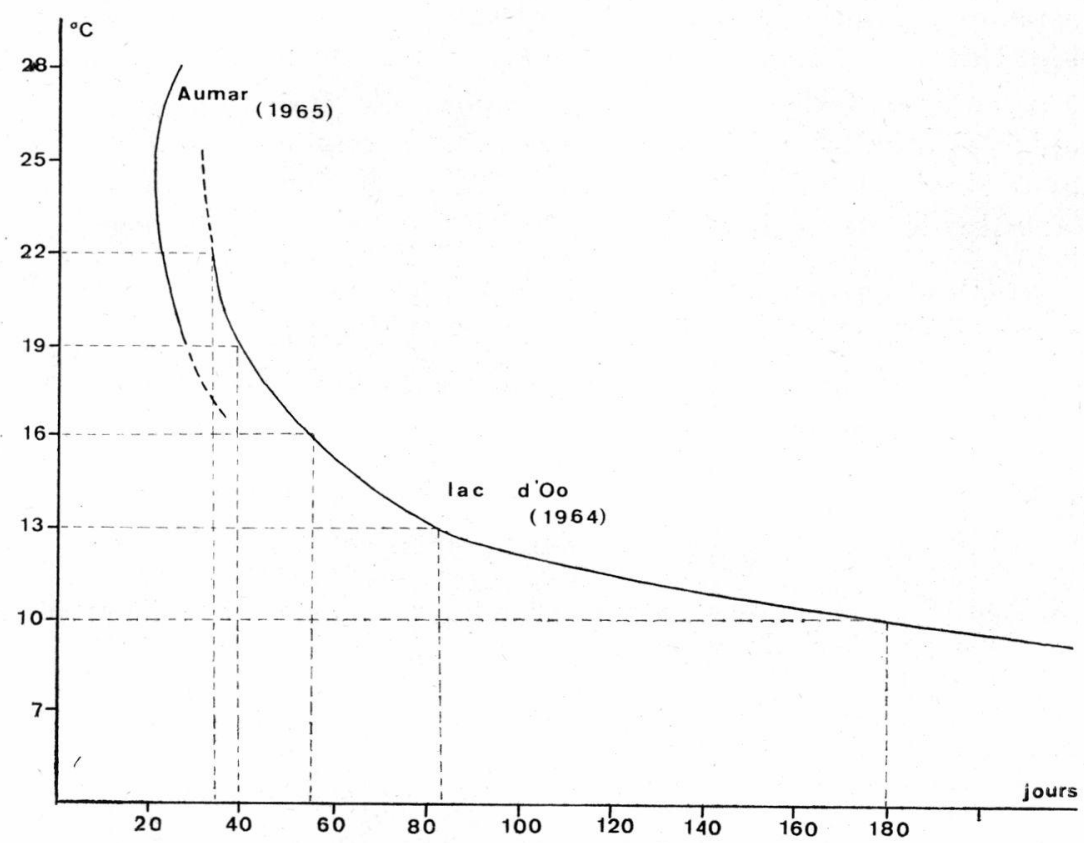

Fig. 2. - Durée de la vie larvaire de Rana temporaria en fonction de la température. 
La courbe de la figure 2 représente cette variation en fonction de la température. En fait, nous obtenons 2 courbes différentes; l'une pour l'année 1964 et l'autre pour l'année 1965. Des facteurs autres que la température sont par conséquent intervenus.

On peut penser à la durée du jour. En effet, le développement des têtards s'est déroulé, — en 1964, — du 24 avril au 27 mai à la température de $22^{\circ} \mathrm{C}$, par exemple. En 1965 , l'élevage à même température s'est déroulé du 5 au 30 juin (durant les jours les plus longs de l'année). Les travaux concernant l'action de la lumière sur le développement (cf. R. GuYÉtant, 1964) appuient cette hypothèse.

On ne peut exclure d'autres facteurs, - notamment la possibilité de races à Constante thermique plus faible, et par conséquent adaptées aux lacs de très haute altitude. En 1964, les œufs de Rana temporaria avaient été récoltés à $1500 \mathrm{~m}$ d'altitude; en 1965, ils venaient d'une toute autre région (Massif du Néouvielle), à $2200 \mathrm{~m}$.

\section{4. - Température létale.}

A $31^{\circ} \mathrm{C}$, les têtards meurent en 2 à 4 jours au plus, à n'importe quel stade de leur développement. A 29 et $30^{\circ} \mathrm{C}$, quelques individus seulement atteignent la métamorphose, et les données concernant la durée de la vie larvaire sont aberrantes.

$28^{\circ} \mathrm{C}$ paraît constituer la limite supérieure de température permettant le développement larvaire de Rana temporaria.

\section{5. - Température seuil et Constante thermique.}

A partir des données expérimentales de 1964, nous pouvons tirer :

$\left(\mathrm{T}-\mathrm{T}_{\mathrm{s}}\right) \mathrm{N}=\left(10-\mathrm{T}_{\mathrm{s}}\right) 170=\left(13-\mathrm{T}_{\mathrm{s}}\right) 83$

$=\left(16-\mathrm{T}_{\mathrm{s}}\right) 55=\left(19-\mathrm{T}_{\mathrm{s}}\right) 41=\left(22-\mathrm{T}_{\mathrm{s}}\right) 33$.

Ces égalités permettent de calculer la température seuil, $7^{\circ} \mathrm{C}$, - et la Constante thermique, - voisine de 500.

En 1965, la Constante thermique est évidemment plus faible, 400 environ.

Le développement larvaire de Rana temporaria est donc possible entre 7 et $28^{\circ} \mathrm{C}$, soit dans des limites supérieures de $3^{\circ} \mathrm{C}$ à celles du développement embryonnaire. La Constante thermique est d'environ 400 ou 500 , suivant des conditions écologiques autres que la température, - ou bien suivant l'origine des oufs.

A la suite de ses propres expériences et de celles de F. Kopscr [1952], E. Balcells [1956] indique une Constante thermique de 830 à 845 pour une température seuil de $2^{\circ} \mathrm{C}$.

Cette température seuil est sujette à caution; elle résulte en effet des observations de J. S. Mikulski [1938], selon lesquelles 
le développement embryonnaire de Rana temporaria serait possible à une température moyenne de $8^{\circ} \mathrm{C}$, avec des oscillations de $\pm 6^{\circ} \mathrm{C}$. Or, nous avons déjà noté le manque de précision des expériences de J. S. Mukulski, qui n'avaient pas permis de calculer la Constante thermique de l'embryon.

Un développement possible à $8^{\circ} \mathrm{C}, \pm 6^{\circ} \mathrm{C}$, ne signifie pas que la température seuil soit de $2^{\circ} \mathrm{C}$. D'autre part, la température seuil de l'embryon et celle du têtard sont différentes.

Si nous reprenons les calculs de BaLcells, mais avec une température seuil de $7^{\circ} \mathrm{C}$, nous obtenons :

$\mathrm{C}^{\mathrm{to}^{\mathrm{t}}}$ thermique $=(16-7) 59=531$

(d'après les expériences de Kopsch)

$\mathrm{C}^{\text {to }}$ thermique $=(17-7) 56=560$

(d'après les expériences de BaLcELLS).

Ces deux valeurs confirment nos expériences de l'année 1964. En effet, les durées de vie larvaire que nous avons indiquées s'entendent du stade A, aux branchies operculées, au stade J. Elles sont inférieures de quelques jours $\left(4\right.$ à $\left.16^{\circ} \mathrm{C}\right)$ à celles observées par Kopsch et Balcells (durée de la vie larvaire depuis l'éclosion jusqu'au stade $\mathbf{J}$ ).

\section{6. - Influence de la température sur la longueur des têtards.}

Les courbes de la figure 3 représentent l'accroissement de la longueur des têtards, en fonction du temps, à différentes températures. La longueur maximale est atteinte au stade $\mathbf{H}$.

La pente des courbes augmente avec la température, ce qui traduit une accélération de la croissance. Par contre, les longueurs maximales, au stade $\mathrm{H}$, diminuent lorsque la température s'élève.

La température a une influence à la fois sur la croissance des têtards et sur leur vitesse de métamorphose (dont rend compte la durée de la vie larvaire). Mais, lorsque la température s'élève, la métamorphose s'accélère à un rythme plus rapide que la croissance.

La longueur maximale des têtards est par conséquent d'autant plus réduite que la durée de la vie larvaire est plus courte : $45,9 \mathrm{~mm}$ à $10^{\circ} \mathrm{C} ; 34,9 \mathrm{~mm}$ à $22^{\circ} \mathrm{C}$. La longueur des jeunes grenouilles varie, dans les mêmes conditions, entre 11 et $17 \mathrm{~mm}$.

Notons que les têtards provenant du Plateau d'Aumar ont une taille nettement plus grande que ceux du lac d'Oô.

Aux températures de 7 et $10^{\circ} \mathrm{C}$, la courbe de croissance, en forme de $\mathrm{S}$, montre que la taille des têtards atteint une limite lorsque la durée de la vie larvaire s'accroît. Nous avons déjà signalé [E. et M.-L. Angelier, 1964] l'achèvement de la croissance 
au stade D, chez une population d'Alytes obstetricans vivant dans un lac de très haute altitude; ils pouvaient demeurer jusqu'à 20 ans à l'état de têtard.

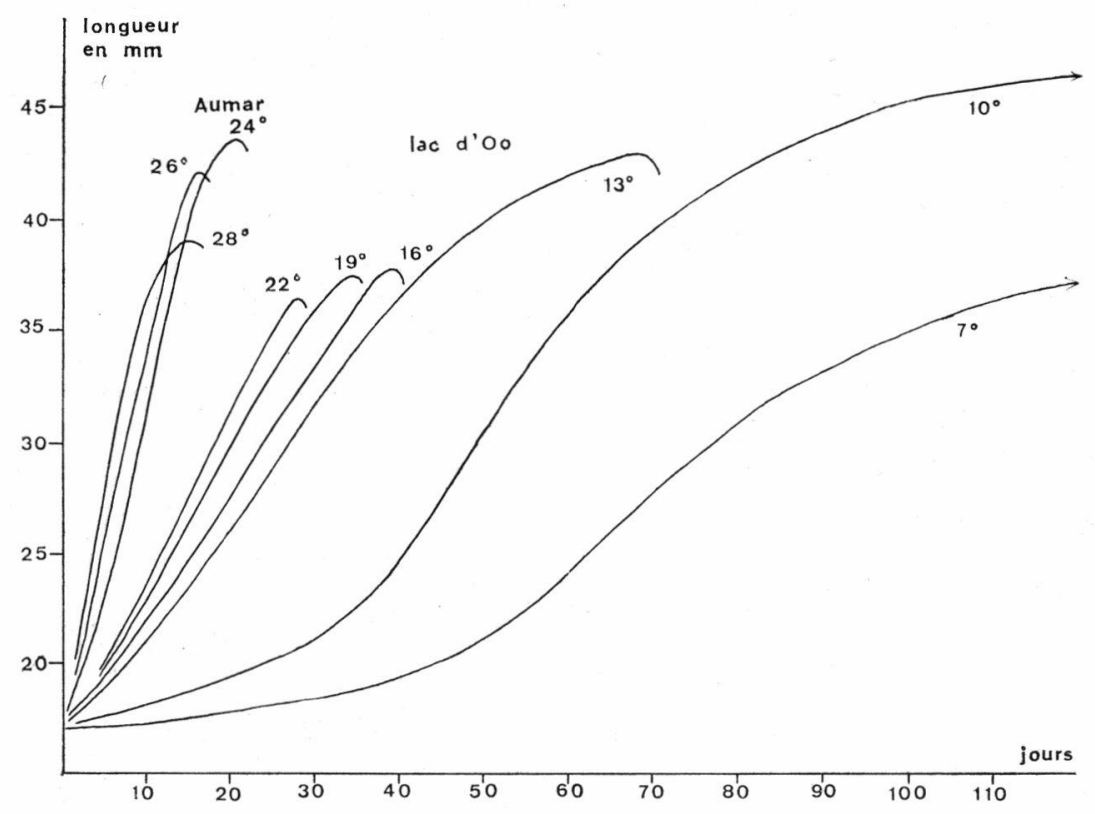

FIg. 3. - Courbes représentant la croissance des têtards de Rana temporaria à différentes températures (du stade $\mathrm{A}$ à $\mathrm{H}$ ).

\section{7. - Température optimale de développement.}

Si la répartition géographique de Rana temporaria nous incite à considérer cette espèce comme sténotherme, l'étude des biotopes des têtards, en montagne, ne nous renseigne pas sur leurs conditions optimales de développement. En effet, la ponte se déroule le plus souvent au hasard des points d'eau formés lors de la fonte des neiges; si les conditions de température conviennent mal aux têtards, ils n'ont aucune possibilité de quitter le biotope où ils sont nés.

Au cours de nos expériences, nous avons remarqué les écarts considérables que présentaient les individus d'un même aquarium, - tant en ce qui concerne la longueur que le stade de développement, - lorsque la température de l'eau tendait vers les valeurs limites.

Lorsque la longueur moyenne des têtards avait atteint sa valeur maximale, dans chaque élevage, nous avons calculé la fluctuation 
de la population autour de la moyenne (variance). Cette fluctuation est donnée par la formule :

$$
\mathrm{F}=\frac{\Sigma(\mathrm{L} m-\mathrm{L} i)^{2}}{\mathrm{~N}-1}
$$

où $\mathrm{Lm}=$ longueur moyenne de la population

$\mathrm{Li}=$ longueur de chaque individu.

$\mathrm{N}=$ nombre d'individus de la population.

La courbe représentant la variation de la fluctuation en fonction de la température (fig. 4), pour les têtards au terme de leur vie larvaire, est en forme de cloche inversée. La fluctuation de la population autour de la longueur moyenne passe par un minimum égal à 4,13 (soit un écart-type, $-\checkmark$ de la fluctuation, de 2,03) à la température de $16^{\circ} \mathrm{C}$.

Cette homogénéité de la population traduit, à notre avis, des conditions optimales de développement. Elle se retrouve dans la métamorphose, - tous les individus devenant adultes à peu près en même temps. Lorsque la température s'élève, la fluctuation de la population augmente rapidement; elle atteint une valeur de 16 (soit un écart-type de 4 ) à $28^{\circ} \mathrm{C}$. Parallèlement, la métamorphose de la population s'étale sur une très longue période. Les premières grenouilles métamorphosées sont plus petites que celles métamorphosées tardivement.

Les mêmes phénomènes s'observent aux basses températures. Mais, à 7 et même $10^{\circ} \mathrm{C}$, la croissance des têtards s'est arrêtée

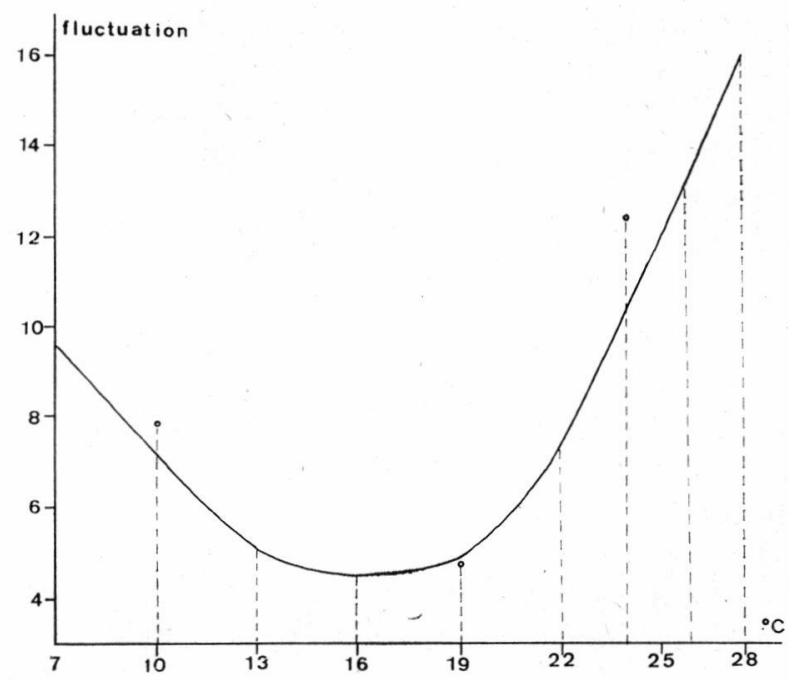

Fig. 4. - Courbe de la fluctuation des têtards de Rana temporaria autour de la longueur moyenne (au stade $\mathrm{H}$ ), en fonction de la température. 
avant les stades ultimes de la métamorphose; il n'y a donc plus de différence de taille entre les premières et les dernières grenouilles sorties de l'eau.

D'autres phénomènes que l'augmentation de la fluctuation incitent à placer à un niveau relativement bas la température optimale de développement des têtards de Rana temporaria: au-delà de $22^{\circ} \mathrm{C}$, on observe fréquemment des malformations de la quene, qui présente des plis verticaux; il en résulte des pcrturbations dans la nage du têtard.

A partir de $26^{\circ} \mathrm{C}$, et jusqu'à la température létale, la durée de la vie larvaire s'accrôit légèrement (fig. 2). Ce fait doit évidemment être noté, mais sans y attacher une importance exagérée : l'étalement de la période de métamorphose est tel, à ces températures (il peut s'écouler jusqu'à 2 mois entre la première et la dernière métamorphose), que le calcul de la durée moyenne de vie larvaire n'a plus guère de signification.

Enfin, une observation intéressante, mais dont nous ne comprenons pas le sens à l'heure actuelle. Le rapport $\frac{\text { Longueur totale } \times 100}{\text { Longueur du tronc }}$ des têtards, lorsqu'ils atteignent leur taille maximale, varie en fonction de la température. La courbe de cette variation (fig. 5) passe par un maximum à $16^{\circ} \mathrm{C}$ (rapport égal à 315 ); c'est précisément la température à laquelle la population est la plus homogène.

\section{8 - Influence des températures alternées sur le développement.}

Nous avons tenté de reproduire, au Laboratoire, les amplitudes quotidiennes de température observées dans les mares de faible profondeur. Une série d'élevages a été réalisée, dont la température maximale atteignait $28^{\circ} \mathrm{C}$, de $5 \mathrm{~h}$ à $21 \mathrm{~h}$ (soit $16 \mathrm{~h}$ par jour). De $21 \mathrm{~h}$ à $5 \mathrm{~h}$, la température était abaissée à 10,16 et $22^{\circ} \mathrm{C}$. Le temps de montée et de descente de la température, pour chaque aquarium, était de $2 \mathrm{~h}$ environ.

Afin de comparer le développement des têtards soumis à de telles amplitudes avec celui de lots témoins à température constante, nous avons calculé la moyenne journalière dans les aquariums à température alternée de la façon suivante :

$16 \mathrm{~h}$ à $28^{\circ} \mathrm{C}$ (soit $448^{\circ} / \mathrm{h}$ ) $+8 \mathrm{~h}$ à $10^{\circ} \mathrm{C}$ (soit $80^{\circ} / \mathrm{h}$ ) $=528^{\circ} \mathrm{C}$ sur $24 \mathrm{~h}$, - soit une moyenne de $22^{\circ} \mathrm{C}$.

Nous obtenons ainsi, pour nos 3 expériences :

\begin{tabular}{|c|c|c|c|}
\hline & A & B & C \\
\hline $5 \mathrm{~h}$ à $21 \mathrm{~h}$ & $28^{\circ} \mathrm{C}$ & $28^{\circ} \mathrm{C}$ & $28^{\circ} \mathrm{C}$ \\
\hline $21 \mathrm{~h}$ & $10^{\circ} \mathrm{C}$ & $16^{\circ} \mathrm{C}$ & $22^{\circ} \mathrm{C}$ \\
\hline $\begin{array}{l}\text { Lots témoins } \\
\left(t^{\circ} \text { moyenne }\right)\end{array}$ & $22^{\circ} \mathrm{C}$ & $24^{\circ} \mathrm{C}$ & $26^{\circ} \mathrm{C}$ \\
\hline
\end{tabular}




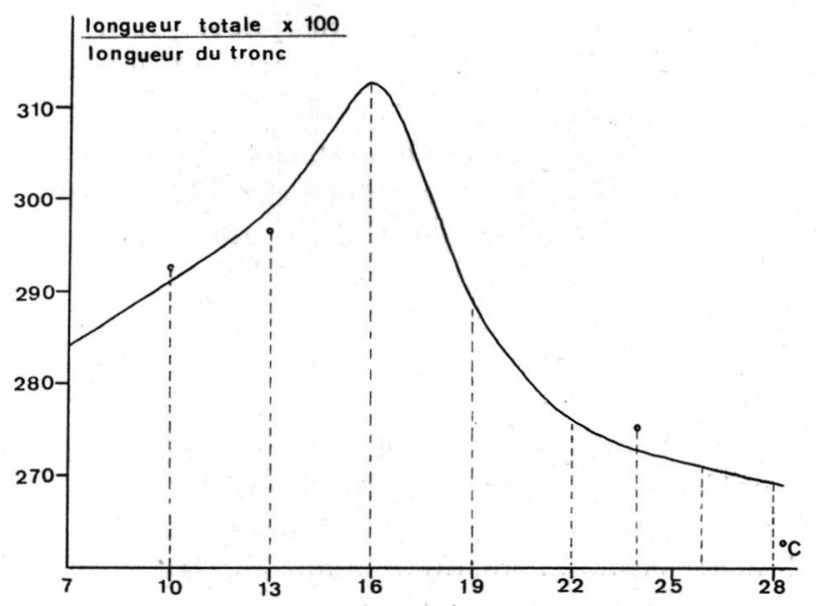

Fig. 5. - Variation du rapport $\frac{\text { Longueur totale } \times 100}{\text { Longueur du tronc }}$ (au stade $H$ ), en fonction de la température.

Après 18 jours d'élevage, les longueurs moyennes des têtards ont été comparées; elles étaient de :

$40,5 \mathrm{~mm}$ pour le lot à $28 / 10^{\circ} \mathrm{C}$ (lot témoin : $46,7 \mathrm{~mm}$ )

$40,7 \mathrm{~mm}$ pour le lot à $28 / 16^{\circ} \mathrm{C}$ (lot témoin : $45,8 \mathrm{~mm}$ )

$38 \mathrm{~mm}$ pour le lot à $28 / 22^{\circ} \mathrm{C}$ (lot témoin : $44 \mathrm{~mm}$ ).

Les milieux à forte amplitude quotidienne de température sont par conséquent défavorables aux têtards de Rana temporaria. Leur croissance subit un retard d'environ 12 à $13 \%$ par rapport à celle de têtards élevés à température constante. La métamorphose subit un retard identique.

Cette absence d'adaptation aux fortes amplitudes de température semble être un des caractères des espèces sténothermes.

\section{4. - DENSITÉ DES TETARDS ET DEVELOPPEMENT LAR- VAIRE.}

D'autres facteurs que la température interviennent évidemment dans le développement larvaire de Rana temporaria, et certains ont déjà fait l'objet de travaux (sels dissous, lumière...).

P. Rотн [1955] avait noté, chez quelques Batraciens, l'existence d'un rythme saisonnier, indépendant des facteurs externes; il correspondait à un arrêt de développement en hiver, même lorsque les conditions de température sont favorables. Or, dans un élevage à $10^{\circ} \mathrm{C}$, les têtards étaient arrivés au stade $\mathrm{H}$ ou $\mathrm{I}$ au début octobre; une partie s'est métamorphosée en fin octobre (vie larvaire : 
170 jours); le reste de la population n'est devenı adulte qu'en mai suivant.

S. M. R. Rose [1961], G. C. Akin [1966] ont mis en évidence une auto-inhibition du développement des têtards, par des substances exsudées, chez Rana pipiens. Nous avons recherché, dans une série d'expériences, l'existence possible d'un tel phénomène chez Rana temporaria.

Nous avons tout d'abord étudié l'influence de la densité des têtards sur le développement, dans une eau régulièrement renouvelée (tous les 3 jours) et constamment filtrée sur charbon. Dans 3 aquariums, à la température de $22^{\circ} \mathrm{C}$, nous avons mis en élevage 20 , 40 et 80 individus, pour 17 litres d'eau.

Au $15^{\circ}$ jour, les longueurs moyennes des têtards sont respectivement de :

$41,6 \mathrm{~mm}(20$ ind $/ 17$ l); $40 \mathrm{~mm}$ (40 ind/17 1); 38,5 mm (80 ind/ $171)$.

Au $20^{\mathrm{e}}$ jour, les $3 / 4$ des têtards sont au stade I dans l'aquarium à plus faible densité; la moitié dans l'aquarium à 40 ind/17 l; aucun dans l'aquarium à 80 ind/17 1 (stade $\mathrm{G}$ et $\mathrm{H}$ ).

Il y a donc peu de différences véritablement significatives entre le développement des têtards, pour une densité de 20 et 40 individus dans 17 litres d'eau. Par contre, le développement est légèrement retardé dans l'aquarium contenant 80 individus.

Nous avons recherché ensuite l'action d'une substance excrétée par les têtards.

En avril 1966, nous avons placé plusieurs pontes recueillies près du lac d'Oô dans un aquarium de 150 litres. La densité des têtards était très élevée, l'eau constamment filtrée sur charbon, mais non renouvelée. Cette eau a été ensuite utilisée pour une série d'expériences; pour plus de clarté, nous la nommerons eau polluée, bien que le terme soit inexact.

Une ponte récoltée en juin, près de l'étang des Nymphes, a été répartie en plusieurs lots, à la température de $22^{\circ} \mathrm{C}$; chaque lot comprenait 40 individus pour 17 litres d'eau.

a) Un lot a été placé dans une eau renouvelée régulièrement.

b) Des œufs, en eau polluée, se sont développés normalement. Mais les jeunes sont morts peu après l'éclosion.

c) Des têtards élevés en eau polluée, après operculation des branchies, subissent un retard considérable de croissance. Leur longueur moyenne n'est que de $\mathbf{2 8 , 9} \mathrm{mm}$ (stade $\mathrm{C}$ du développement) lorsque celle du lot témoin est de $41,6 \mathrm{~mm}$ (stades E-F).

d) Des têtards élevés dans une eau pure, puis transférés au stade $\mathbf{E}$ en eau polluée, ne subissent plus de retard appréciable du développement. 
e) Des têtards élevés en eau polluée, puis transférés en eau pure, renouvelée, reprennent à peu près leur vitesse de croissance normale.

Ces observations, qui doivent être considérées comme préliminaires, confirment celles de Rose et AKıN chez Rana pipiens.

\section{5. - CONCLUSIONS.}

Le but de nos recherches était de comprendre les phénomènes observés dans les lacs et mares de haute montagne, concernant le développement de Rana temporaria.

Les températures limites sont de 4 et $25^{\circ} \mathrm{C}$ pour le développement embryonnaire, -7 et $28^{\circ} \mathrm{C}$ pour le développement larvaire.

La Constante thermique des têtards, issus de pontes recueillies à $2200 \mathrm{~m}$ et élevés en juin (400), est plus faible que celle des têtards élevés en avril-mai à partir de pontes récoltées à $1500 \mathrm{~m}$ $(500)$. Ceci traduit l'influence de facteurs non contrôlés (lumière), ou la possibilité de races adaptées à la haute montagne.

Le rythme de la métamorphose s'accélère plus rapidement, avec la température, que celui de la croissance. La taille des jeunes grenouilles est par conséquent plus faible lorsque la durée de la vie larvaire diminue. Ceci explique la présence de grenouilles naines dans les mares temporaires, - où seuls les individus les plus précoces ont le temps de se métamorphoser avant l'assèchement.

La fluctuation de la population autour de la longueur moyenne est minimale à $16^{\circ} \mathrm{C}$; elle s'accroît lorsque la température diminue, et plus encore lorsqu'elle augmente. L'homogénéité de la population correspond sans aucun doute à des conditions optimales de développement. Les têtards du lac Supérieur d'Estibère, — à $2330 \mathrm{~m}$ d'altitude, - se développent dans ces conditions optimales, car la température ne dépasse pas $16-18^{\circ} \mathrm{C}$ en été. La métamorphose de l'ensemble de la population se déroule en quelques jours.

Dans les lacs à température élevée en été (lac Inférieur d'Estibère, à $2120 \mathrm{~m}$ ), l'étalement de la période de métamorphose est considérable (de fin juillet à début septembre). Les premières grenouilles métamorphosées sont de petite taille $(11 \mathrm{~mm})$; les dernières atteignent $17 \mathrm{~mm}$.

Les fortes amplitudes de température qu'on observe dans les mares de faible profondeur sont défavorables au développement des têtards. Ceci confirme le caractère sténotherme de Rana temporaria.

Enfin, une série d'expériences préliminaires a permis de mettre en évidence le rôle de la densité de la population et des substances excrétées par les têtards sur le développement larvaire. Mais, en 
dehors de très petites mares, il ne semble pas que ce facteur puisse avoir une importance dans les biotopes étudiés.

\section{RÉSUMÉ}

Les auteurs ont étudié la durée du développement embryonnaire et de la vie larvaire de Rana temporaria dans les lacs et mares de haute altitude des Pyrénées centrales. Cette durée, - et aussi la longueur de la jeune grenouille après la métamorphose - varient considérablement en fonction de l'altitude. Des élevages à différentes températures ont été réalisés pour expliquer ce phénomène.

Le développement de l'œuf se déroule entre $4^{\circ}$ et $25^{\circ} \mathrm{C}$, et sa durée varie entre 3 et 20 jours. La constante thermique est de 60 environ.

Le développement du têtard s'effectue entre $7^{\circ}$ et $28^{\circ} \mathrm{C}$. Sa durée varie, en fonction de la température, de 20 jours $\left(28^{\circ} \mathrm{C}\right)$ à plus de 18 mois. La constante thermique est de 500 environ.

La longueur du têtard, à la fin de sa vie larvaire, et celle de la jeune grenouille, est plus faible lorsque la vitesse de développement augmente.

La fluctuation autour de la longueur moyenne du têtard, à la fin de la vie larvaire, a été calculée. La courbe de cette fluctuation, en fonction de la température, passe par un minimum à $16^{\circ} \mathrm{C}$. Les auteurs pensent que l'homogénéité de la population à cette température traduit des conditions optimales de développement.

D'autres facteurs que la température interviennent dans la durée de la vie larvaire. Des œufs récoltés à $2200 \mathrm{~m}$ d'altitude, et mis en élevage en juin, se développent plus rapidement, — à température égale — que des ceufs récoltés en avril à $1500 \mathrm{~m}$.

\section{OBSERVATIONS ON THE EMBRYONIC AND LARVAL DEVELOPMENT OF RANA TEMPORARIA}

[Amphibia, Salientia ]

The authors have studied the duration of the embryonic development and of larval life for Rana temporaria in the lakes and ponds at high altitude in the central Pyrenees. This duration, and also the length of the young frog after metamorphosis, - - vary considerably according to the altitudes. Rearing at different temperatures was carried out to explain this phenomenon.

The development of the egg takes place between $4^{\circ} \mathrm{C}$ and $25^{\circ} \mathrm{C}$, and its duration varies between 3 and 20 days. The thermal constant is about 60 .

The development of the tadpole occurs between 7 and $28^{\circ} \mathrm{C}$. The duration varies according to the temperature from 20 days (at $28^{\circ} \mathrm{C}$ ) to about 18 months. The thermal constant is about $\mathbf{5 0 0}$.

The length of the tadpole at the end of the larval stage, and that of the young frog, is less important when the speed of development increases.

The fluctuation around the medium length of the tadpole at the end of the larval stage has been calculated. The curve of this fluctuation, according to the temperature, touches a minimum of $16^{\circ} \mathrm{C}$. The 
authors think that the homogeneity of the population at this temperature is explained by optimal conditions of development.

Other factors besides the temperature affect the duration of the larval stage. Eggs collected at $2200 \mathrm{~m}$ and reared in June, develop more rapidly - at the same temperature - than eggs collected in April at $1500 \mathrm{~m}$.

\section{BEOBACHTUNGEN ÜBER DIE ENTWICKLUNG BEIM EMBRYON UND BEI DER LARVE VON RANA TEMPORARIA \\ [Amphibia, Salientia]}

Die Entwicklungsdauer des Embryons und das Leben der Larven von Rana temporaria aus den Seen und Tümpel des Hochgebirges der Zentralpyrenäen sind untersucht worden. Diese Dauer ebenso wie die Grösse des jungen Frosches nach der Metamorphose, sind sehr stark von der Höhenlage abhängig. Um diese Tatsache zu erklären durchgeführt worden.

Die Entwicklung des Eies findet zwischen $4^{\circ}$ und $25^{\circ} \mathrm{C}$ statt und ihre Dauer schwankt zwischen 3 einhalb und 20 Tagen. Die thermische Konstante beträgt umgefähr 60 .

Die Entwicklung des Lurches finclet zwischen $7^{\circ}$ und $28^{\circ} \mathrm{C}$ statt. Die Dauer schwankt in Abhängigkeit von der Temperatur von 20 Tagen, bei $28^{\circ} \mathrm{C}$, bis zu fast 18 Monaten. Die thermische Konstante beträgt ungefähr 500 .

Die Grösse des Lurches, am Ende des Larvenstadiums, und die des jungen Frosches ist um so geringer, desto schneller die Entwicklung vor sich geht.

Schwankungen rund um den Mittelwert der Grösse des Lurches, am Ende des Larvenstadiums sind berechnet worden.

In Funktion von der Temperatur geht diese Schwankungskurve bei $16^{\circ} \mathrm{C}$ durch ein Minimum. Die Autoren nehmen an, dass die Homogenität der Bevölkerung bei dieser Temperatur den optimalen Entwicklungsbedingungen entspricht.

Ausser der Temperatur spielen noch weitere Faktoren bei den Entwicklung des Larvenstadium eine Rolle. Bei gleicher Temperatur entwickeln sich Eier die im Juni in $2200 \mathrm{~m}$ Höhe gesammelt und anschliessend im Labor gezüchtet wurden viel schneller als Eier die im April in $1500 \mathrm{~m}$ Höhe entnommen wurden.

\section{TRAVAUX GITES}

Akın (G. C.). 1966. - Self-inhibition of growth in Rana pipiens tadpoles. Physiol. Zool., U.S.A., 39, 4 : 341-356.

ANGer (E.). 1946. - Amphibiens et Reptiles. Faune de France, Lechevalier, Paris, 204 pp.

ANGelier (E.). 1961. - Les lacs de l'Estibère : remarques sur le peuplement des lacs de montagne. Bull. Soc. Hist. Nat. Toulouse, 96 : 7-14.

ANGetier (E. et M.-L.). 1964. - Etude d'une population de Crapaud accoucheur dans un lac de haute montagne. C. R. Acad. Sci., 258 : 701-703.

Balcells (E.). 1956. - Estudio morfologico, biologico y ecologico de Rana temporaria L. Bull. Inst. Biol. apl., Barcelona, 24 : 81-103. 
Beck (P.). 1943. - Note préliminaire sur la faune herpétologique des Hautes-Pyrénées. Bull. Soc. Sci. Htes-Pyrénées, 1 : 48-58.

BeER (A.). 1956. - Der Einfluss der Dunkelheit auf der Wachstum und die Metamorphose der Kaulkappen von Xenopus laevis. Z. Vitam. Horm. u. Fermentforschg., 8,2/3 : 86-97.

Combes (Cl.). 1967. - Biologie, écologie des cycles et biogéographie de Digènes et Monogènes d'Amphibiens dans l'Est des Pyrénées. Thèse, Montpellier, $432 \mathrm{p}$.

Doetsch (H.). 1949. - Experimentelle Untersuchungen über Einfluss des Lichtes und der U.V. Strahlen auf Wachstum unt Entwicklung von Amphibienlarven. Wilhelm-Roux'Arch., 144, 1 : 25-30.

Dougras (R.). 1948. - Temperature and rate development of the eggs of british Anura. J. anim. Ecol., 17 : 189-192.

Gall.ien (L.). 1941. - Sur une race différenciée de Rana temporaria de la région pyrénéenne. Bull. Soc. Zool. Fr., $66: 80-84$.

GuyÉtant (R.). 1964. - Actions comparées de la lumière et de l'obscurité sur la croissance et la métamorphose des têtards de Rana temporaria. - Note préliminaire. Ann. Sci. Univ. Besançon,

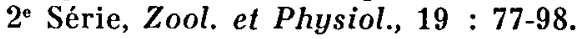

Gưḱtant (R.). 1966. — Observations écologiques sur les pontes de Rana temporaria dans la région de Besançon. Ann. Sci. Univ. Besançon, Physiol, et Biol. anim., $3^{\mathrm{e}}$ Série, 2 : 12-18.

Inikнmovitch (A. I.). 1947. - Influence de la lumière sur la vitesse de développement des têtards de Rana temporaria (en russe). Dokl. Akad. Nauck. SSSR, 55, 2 : 177-180.

Kaunr (H.). 1955. - Das Licht als hemmenden Faktor bei der Larventwicklung des Wechselkröte. Oikos, 6, 1 : 78-91.

Kopsch (F.). 1952. - Entwicklung des braunen Grasfroschen Rana fusca. G. Thieme Verlag, Stuttgart, $70 \mathrm{p}$.

Mrkulski (J. S.). 1938. - Experimental sudies in thermal ecology of developing eggs of some amphibians (Rana, Bufo, Pelobates). Acad. Pol. Sci. Let., Cracovie, Série B, 2 : 23-45.

Moore (J. A.). 1939. - Temperature tolerance and rates of development in the eggs of Amphibia. Ecology, 20 : 459-478.

Moore (J. A.). 1951. - Hibridazion and embryonic temperature adaptation studies of Rana temporaria and R. sylvatica. Proc. Nat. Acad. Sci., $37: 862-868$.

Rose (S. M. R.) et Rose (F. C.). 1961. - Growth controlling exudates of tadpoles. Symposia Soc. Exper. Biol., 15 (Mechanism in Biological competition), Cambridge : 207-218.

Roth (P.). 1955. - Les métamorphoses des Batraciens. Dunod édit., Paris, 98 p.

RüHMEKoRF (E.). 1958. - Beiträge zur ökologie mitteleuropäischer Salienta. III. Wirkungen des Lichtes auf Laich und Larven. $\boldsymbol{Z}$. Morphol. okol. Tiere, 47, 1 : 37-53.

Savage (R. M.). 1961. - The ecology and life history of the Common frog (Rana temporaria L.). Pitman, London, 221 p.

VAllée (L.). 1947. - Contribution à l'étude des races sexuelles françaises de Rana temporaria L. Bull. Soc. Zool. Fr., 77, 2 : 97-100.

VAN DER Eeckhoudt (J. P.). 1949. - Recherches sur l'influence de la lumière sur la métamorphose de Grenouille rousse. Ann. Soc. $r_{\text {c }}$ Zool. Belg., 80 : 40-75.

(Laboratoire d'Hydrobiologie de la Faculté des Sciences de Toulouse, Equipe de recherches associée au C.N.R.S.) 